\title{
Le mensonge féminin comme principe libérateur. D’Irmtraud Morgner à Brigitte Burmeister : passage de témoin
}

Weibliche Lüge als Befreiungsstrategie. Von Irmtraud Morgner zu Brigitte

Burmeister: Ein Paradigmentransfer

Woman's deceit as liberation strategy. The passing of the baton from Irmtraud

Morgner to Brigitte Burmeister

\section{Catherine Teissier}

\section{OpenEdition}

Journals

Édition électronique

URL : http://journals.openedition.org/ceg/1537

DOI : $10.4000 /$ ceg. 1537

ISSN : 2605-8359

\section{Éditeur}

Presses Universitaires de Provence

Édition imprimée

Date de publication : 15 juin 2015

Pagination : 177-190

ISBN : 978-2-85399-993-9

ISSN : 0751-4239

\section{Référence électronique}

Catherine Teissier, « Le mensonge féminin comme principe libérateur. D'Irmtraud Morgner à Brigitte Burmeister : passage de témoin », Cahiers d'Études Germaniques [En ligne], 68 | 2015, mis en ligne le 17 décembre 2017, consulté le 02 décembre 2020. URL : http://journals.openedition.org/ceg/1537 ; DOI : https://doi.org/10.4000/ceg. 1537 


\section{Le mensonge féminin comme principe libérateur. D'Irmtraud Morgner à Brigitte Burmeister: passage de témoin}

Catherine TEISSIER Aix Marseille Université

Selon la définition de l'anthropologue Maurice Godelier, « ce que l'on appelle genre, masculin ou féminin, est l'ensemble des attributs qu'une société attache aux individus selon qu'ils sont homme ou femme à la naissance ${ }^{1}$ »). Or, les sociétés assignent également une position différente dans les rapports sociaux, notamment dans les rapports de pouvoir, à l'individu selon qu'il est masculin ou féminin (ce que Françoise Héritier appelle la «valence différentielle des $\operatorname{sexes}^{2}$ »). Les rapports de pouvoir sont des rapports construits socialement, mais justifiés par la catégorie du sexe. Ce que M. Godelier formule de la manière suivante: «Les rapports sociaux s'enfouissent donc dans le corps sexué des individus qui fonctionne comme une sorte de "machine ventriloque" de l'ordre qui règne dans la société ${ }^{3}$.» Si l'on ajoute que les représentations que se fait une société donnée de l'attribution des pouvoirs à tel ou tel individu selon son sexe ne sont pas inconscientes, mais qu'elles font au contraire partie de la conscience sociale, collective et individuelle, et qu'elles sont jusqu'à un certain point partagées par les dominants et les dominés, on comprend que la mise en cause de ces représentations soit une question complexe.

Dans le roman de Brigitte Burmeister, Unter dem Namen Norma (Sous le nom de Norma), paru en 1994 et non traduit en français à ce jour ${ }^{4}$, la domination liée au genre en croise une autre, celle subie par les vaincus de l'histoire. Marianne, la narratrice autodiégétique, et la plupart des personnages du roman, font en effet partie des perdants de la Réunification allemande, ceux dont le monde, les repères, le passé disparaît tout en perdant toute légitimité, puisque marqué par l'opprobre de la dictature. Unter dem Namen Norma est aussi un roman sur la perte d'identité, comme cela a déjà été montré 5 .

1 Maurice Godelier, «Femmes, sexe ou genre? », in Margaret MARUANi (dir.), Femmes, genre et sociétés. L'état des savoirs, Paris, La Découverte, 2005, p. 15.

2 Françoise Heritier, Masculin-Féminin, la pensée de la différence, Paris, Odile Jacob, 1996.

3 Godelier, « Femmes, sexe ou genre? », p. 18-19.

4 Brigitte Burmeister, Unter dem Namen Norma, Stuttgart, Klett-Cotta, 1996. Cité dans la suite du texte sous Norma. Toutes les traductions ont été réalisées par nos soins.

5 Henk Harbers, « Die leere Mitte: Identität, Offenheit und selbstreflexives Erzählen » in Brigitte Burmeister, Unter dem Namen Norma, Weimarer Beiträge, 50 (2), 2004, p. 227-241. 
Mais Marianne va résister à cette perte. Doublement réduite au silence, en tant que femme et en tant que membre d'une société qu'on efface, elle va reconquérir sa parole par un mensonge spectaculaire, puisqu'il prend la forme d'une révélation attendue.

Il s'agira ici d'étudier la genèse, les mécanismes et les acteurs d'un mensonge féminin qui est aussi la réponse plus large du dominé est-allemand au dominant de l'ouest et à son modèle de vérité. Auparavant, on aura rappelé que le jeu avec le mensonge et la vérité est une manière de délégitimer le discours de pouvoir, y compris symbolique, et que cette entreprise a été menée dès les années soixante par une écrivaine aujourd'hui un peu oubliée, Irmtraud Morgner. Dans cet article, nous tenterons de replacer le roman de B. Burmeister dans la postérité de cette autre auteure est-allemande, qui a élevé le mensonge féminin au rang de principe émancipateur.

\section{Le mensonge libérateur (I. Morgner)}

L'œuvre foisonnante d'I. Morgner (1933-1990) fait une grande place au mensonge en tant que principe libérateur des groupes historiquement opprimés, comme le prolétariat ou les femmes. Dès les premiers romans, le mensonge apparaît aux protagonistes féminins comme le moyen d'échapper à une réalité insupportable, mais aussi comme la première étape pour inventer une autre réalité. Dans et à partir de Notturno (Nocturne) - récit de 1962 dont une seconde version paraîtra dans le roman censuré Rumba auf einen Herbst (Rumba sur un automne), $1965^{6}$-, il y a deux sortes de mensonges, le mensonge bassement utile d'abord, celui qui sert à supporter la vie. Ce sont les mensonges que Karla, le personnage principal, se fait à elle-même, en usant et abusant des comparaisons, pour supporter sa condition d'épouse et de mère au foyer frustrée. Mais il y a aussi le 'grand' mensonge, le mensonge créatif, inventif, le mensonge qui ouvre les portes d'un autre monde, un monde fabuleux qui constitue la réserve de l'utopie. Le premier mensonge est un pis-aller, le second, une nécessité. L'un des personnages reproche à Karla le mauvais mensonge: « Vous utilisez votre imagination laissée à l'abandon pour tourner le monde à votre façon avec vos mensonges ${ }^{7}$. » Kai, le personnage masculin de Notturno, va plus loin:

Mich kotzt das an. [...] Solche schlechten Lügen kotzen mich an. [...] Du hast früher wunderbar lügen können, zauberhaft, produktiv. Hast du gar keine Phantasie mehr? Musst du sie so pervertieren, dass man glatt auf den Gedanken kommt, du hast keinen Funken mehr davon? ${ }^{8}$

Le mensonge libérateur, qui permet de révéler le caractère aliénant de la réalité vécue mais aussi de prendre sa revanche sur cette réalité, est au cœur du

6 Irmtraud Morgner, Rumba auf einen Herbst, éd. par Rudolf Bussmann, avec une concordance de Doris JaHnsen, Hamburg/ Zürich, Luchterhand Literaturverlag, 1992. Cité par la suite sous: Rumba. Toutes les traductions ont été réalisées par nos soins.

7 «Sie wenden Ihre brachliegende Phantasie auf, um sich die Welt zurechtzulügen.» Rumba, p. 145 .

8 Ibid., p. 211. 
roman suivant et est affiché dès le titre comme son principe d'organisation: Die wundersamen Reisen Gustavs des Weltfahrers. Lügenhafter Roman mit Kommentaren (Les étonnants voyages de Gustave l'explorateur - Roman mensonger avec commentaires, 1972) ${ }^{9}$. Le roman est dit «mensonger» parce que reposant sur un jeu ambigu avec les instances narratives: le jeu avec la forme commence dès la couverture du livre et se poursuit par un jeu d'emboîtements successifs, tels ceux dont s'amusait E.T.A. Hoffmann ${ }^{10}$. Ces histoires mensongères racontées par Gustave l'explorateur forment la première «boîte »: les récits encadrés. Gustave l'explorateur les raconte à Gustave l'éboueur: ce niveau forme le récit-cadre. Mais l'ensemble du dispositif est annoncé par une préface, écrite par la petite-fille de Gustave l'explorateur, Bele H., qui se présente elle-même comme auteure du livre. Celle-ci commente en outre les récits de son grand-père dans des notes de bas de page, ce qui accentue encore la mise en abyme du récit. Enfin, le livre et sa préface sont édités par un autre personnage, Dr. Phil. Beate Heidenreich, liée à plus d'un titre à Bele H., ce qu'elle expose dans une postface: elle se dit son amie, a le même âge, a vécu dans la même maison dans leur enfance, a fréquenté la même école et a les mêmes initiales. Préface et postface donnent donc un second cadre aux récits de Gustave.

Ce redoublement des instances narratives, et grâce à lui, la mise à distance et la remise en question du narrateur des récits encadrés, Gustave l'explorateur (le menteur), ce dispositif, donc, a un effet essentiel, celui de libérer la parole de Gustave l'éboueur - ainsi que la parole féminine. Die wundersamen Reisen Gustavs paraît aux éditions Aufbau-Verlag en 1972, et a sans doute bénéficié de la relative liberté d'expression accordée aux artistes après le discours d'Honecker lors de la $4^{e}$ session du Comité Central de 1971. Pourtant, le roman doit malgré tout subir une modification: l'ajout d'une « Postface de l'éditrice». Il est donc clair que ces réfractions des instances et des voix ont pour but de faire passer le message subversif contenu dans les récits eux-mêmes. Toutefois, l'objectif principal de l'auteure est de donner la parole à ceux qui sont encore réduits au silence, les prolétaires puis les femmes. Et pour cela, le passage par le « mensonge » semble indispensable.

C'est particulièrement le cas dans les récits autour du personnage de la grandmère. La dimension tragique de son destin est double, d'abord parce qu'elle est aliénée en tant que prolétaire et en tant que femme, mais aussi parce que ce destin est oublié, qu'il ne s'inscrit dans aucune mémoire, ni familiale, ni individuelle. C'est par la puissance de l'imagination que Bele va tenter de déjouer l'oubli, par ce qu'elle appelle les « fleurs de mensonge »:

Beispielsweise auf Familienfeiern gesanglich gefassten Wunschäußerungen des Inhalts, noch mal jung sein zu wollen, pflegte meine Großmutter Klara zu erwidern : « Um Gottes willen, das möcht ich nicht noch mal durchmachen. » Nur diese Erwiderung und Haar,

9 Irmtraud Morgner, Die wundersamen Reisen Gustavs des Weltfahrers. Lügenhafter Roman mit

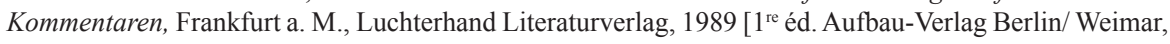
1972]. Cité par la suite sous: Die wundersamen Reisen Gustavs. Toutes les traductions ont été réalisées par nos soins.

${ }^{10}$ E.T.A. Hoffmann, Les Frères de Saint-Sérapion III, éd. par A. Beguin et M. Laval, Paris, Phébus, 1982. 
das auf dem kleinen Kopf lastete wie eine Schneemütze, hat mein Gedächtnis von ihr behalten. Es hat sie expropriiert wie die Familie. Ich kann ihr das Eigentum nicht direkt zurückerstatten. Die Geschichte verzeichnet Sieger, Klara, eine Besiegte, hat vierzehn Kinder geboren, auch den Weltfahrer. Ihrem ausgemergelten Leib entwuchsen und entwachsen noch und endlos lügenhafte Blumen. Ich erwarte die Zeit, da sie nimmt, was ihr gehört. ${ }^{11}$

Dans ces phrases un peu énigmatiques s'exprime nettement la dette que la narratrice ressent envers la grand-mère, et la nature de cette dette, d'ordre physique, poétique, et symbolique. Il n'y a pas de parole, ni de créativité possible, sans l'existence sacrifiée de Klara, mère et grand-mère. La dette ne peut être payée « directement», elle le sera donc indirectement: par la reconnaissance que c'est du corps imaginaire de Klara, que Bele s'approprie, que naissent les «fleurs de mensonge », c'est-à-dire les récits que Bele attribue à Gustave.

La libération d'une parole féminine passe par le mensonge, car les femmes comme les prolétaires, naguère (la RDA des années soixante-dix) comme jadis, ne disposent pas de la position dans l'ordre symbolique qui leur autoriserait une parole directe. Il s'agit donc de ruser, de simuler, afin, comme l'analyse Vladimir Jankélévitch, de « nous induire en vérité par la voie indirecte de la simulation ${ }^{12}$ ». En effet, chez I. Morgner, le mensonge est bien une stratégie, utilisée par le personnage pour résister aux différentes formes de domination, mais aussi pour révéler cette dernière: dans son ouvrage le plus célèbre, Leben und Abenteuer der Trobadora Beatriz nach Zeugnissen ihrer Spielfrau Laura (Vie et aventures de la Trobairitz Béatrice d'après les témoignages de sa ménestrelle Laura, 1974) ${ }^{13}$, I. Morgner retrace les aventures de la Trobairitz Béatrice, plongée dans un sommeil magique en plein Moyen Age pour échapper à son destin, et réveillée par erreur en 1968. Béatrice, par bien des aspects une véritable picara, va traverser la France de l'époque, où l'égalité des sexes n'existe toujours pas, subir toutes les violences faites aux femmes (viol, exploitation sexuelle, injustices de tous ordres) au long de ses mésaventures, pour arriver enfin à la « Terre Promise des femmes », la RDA, où, bien sûr, elle connaîtra là aussi un certain nombre de déceptions.

Pour résister à l'adversité, Béatrice érige le mensonge en principe de son existence. Elle ne renonce jamais à elle-même, mais en bonne picara, résiste par la ruse, le mensonge, la fuite. La force du picaro n'est pas en effet de contrer violemment la pression exercée par autrui, mais de remporter d'imperceptibles victoires dans les interstices de la vie quotidienne. Ne s'avouant jamais battue, Béatrice sait exploiter les possibilités de résistance des faibles, utiliser la dissimulation comme stratégie de survie, s'arranger avec une réalité insupportable: son premier mari par exemple, qui l'exploite sexuellement et matériellement, est cocufié à l'envi. La menteuse prend sa

11 Die wundersamen Reisen Gustavs, p. 87.

12 Vladimir JankéLÉVITCH, Traité des Vertus II, t. 1, «Les vertus et l'amour », Paris, Flammarion, 1986, p. 182. V. Jankélévitch analyse ici la litote, « qui n'est pas menteuse », c'est-à-dire qu'elle n'utilise pas l'intention trompeuse, mais est un outil pour parvenir à la vérité.

13 Irmtraud MoRgner, Leben und Abenteuer der Trobadora Beatriz nach Zeugnissen ihrer Spielfrau Laura. Roman in dreizehn Büchern und sieben Intermezzos, Frankfurt a. M., Luchterhand Literaturverlag, 1989 [1 ${ }^{\text {re }}$ éd. Aufbau-Verlag Berlin/ Weimar, 1974]. Cité par la suite sous: Leben und Abenteuer der Trobadora Beatriz. Toutes les traductions ont été réalisées par nos soins. 
revanche, puisque elle sait la vérité, alors que sa dupe l'ignore. C'est ce qui fait la supériorité du trompeur sur la dupe ${ }^{14}$.

Tout au long de ses voyages dans les pays de l'Ouest, avant, mais aussi après sa découverte de sa «Terre promise », et de sa rencontre avec Laura, l'amie et ménestrelle, Béatrice n'hésite jamais à tromper, à mentir, à se faire passer pour ce qu'elle n'est pas, s'appropriant les écrits de Laura, promettant des livres qu'elle n'écrit pas pour obtenir de l'argent, etc. Quant à Laura, elle interprétera les récits les plus incroyables faits par Béatrice comme la preuve que son imagination se libère peu à peu, lui permettant de remplir sa mission, celle d'une « prophète au féminin ». C'est en particulier le cas pour l'un des principaux récits encadrés du roman, la «Bonne nouvelle selon Valeska », récit d'un changement de sexe, présenté comme la solution pour mettre fin à la domination masculine et à l'oppression des femmes. Cette métamorphose n'est tout d'abord pas prise au sérieux par Laura ${ }^{15}$, qui qualifie le récit de «mensonge ». Mais Laura changera d'avis plus tard dans le roman, lisant cette « Bonne nouvelle » après la mort de la Trobairitz comme une « révélation ${ }^{16}$ ». Cette interprétation est certes relativisée par un certain nombre de marqueurs de l'ironie, en premier lieu l'exagération que représente l'écart extrême entre les deux interprétations, « mensonge » et « révélation ». Reste cependant l'utilisation brillante que fait I. Morgner de différents modes d'expression indirecte («mensonge», " légende », ironie) pour, à travers ses personnages, libérer une position et une parole féminine qui sans cela ne peuvent s'articuler. Le passage par le « mensonge » permet, c'est le postulat de l'auteure, de faire passer en fraude un certain nombre de vérités, de miner les certitudes, de délégitimer le discours du pouvoir.

Ce procédé va se retrouver vingt ans après le roman de 1974, Leben und Abenteuer der Trobadora Beatriz, chez une contemporaine d'I. Morgner, Brigitte Burmeister (née en 1940). Dans le roman Unter dem Namen Norma (1994), la protagoniste connaît une double défaite, elle fait partie des perdants à un double titre: en tant que citoyenne de la défunte $\mathrm{RDA}$, et en tant que femme. Le roman se situe en effet à ce moment charnière des premiers mois de l'année 1990 où tout un monde disparaît, où une société (celle, victorieuse, de l'Allemagne de l'Ouest) en absorbe une autre (celle, perdante, de l'Allemagne de l'Est), lui déniant par la même occasion toute forme de légitimité. Parmi ceux et celles qui voient leur vie se défaire et leur passé devenir douteux, les femmes sont celles qui perdent le plus. Privées de façon massive de leur indépendance économique, elles perdent également leur position dans la société, société qui avait évolué depuis les années soixante-dix et leur laissait à la fin des années quatre-vingt une place d'où parler enfin d'elles-mêmes à égalité avec les hommes. Au début des années 90, ces hommes de l'ancienne RDA sont eux aussi des perdants - mais dans une moindre mesure. En Marianne, la protagoniste du roman de B. Burmeister, s'articulent donc ces deux défaites, ces deux manières d'être symboliquement réduite au silence: en tant que femme et en temps que membre d'une société disparue, d'un

\footnotetext{
14 JANKÉLÉVITCH, « Les vertus et l'amour », p. 183.

15 «Laura hatten die Unglaublichkeiten des Berichts keineswegs ergrimmt. Im Gegenteil, seitdem Beatriz zu Studienzwecken in Betrieben anheuerte, wertete Laura deren Lügen ausnahmslos als Anzeichen schöpferischen Überdrucks. » Leben und Abenteuer der Trobadora Beatriz, p. 421.

${ }^{16}$ «Laura las es am Begräbnistag der Trobadora. Als Offenbarung. » Ibid.
} 
système marqué par l'opprobre. Pour sortir de cette situation et reconquérir sa parole, Marianne va choisir une voie spectaculaire: un mensonge, le pire mensonge qu'on puisse faire à l'époque car il prend la forme d'une révélation attendue.

\section{Le mensonge révélateur (B. Burmeister)}

Le roman de B. Burmeister appartient à ceux que l'on a appelés «WendeRomane », on parle aussi de «Wendeliteratur », romans du Tournant, romans qui tentent de combler ce besoin de comprendre ce qui s'est passé, de rendre compte des immenses bouleversements existentiels, émotionnels, qu'ont vécus les Allemands, et surtout les Allemands de l'Est, après le 9 novembre 1989. Salué à sa sortie comme l'un des meilleurs de ces «Wende-Romane ${ }^{17}$ », Unter dem Namen Norma n'a pas eu le succès d'autres romans du Tournant, tels que Helden wie wir, de Thomas Brussig ${ }^{18}$, ou Simple Storys, de Ingo Schulze ${ }^{19}$. Mais il semble avoir mieux vieilli que d'autres.

Le mensonge est un élément incontournable du Tournant (Wende), cette période allant de l'été 1989 à l'automne 1990 et qui permit le passage sans violence du « socialisme réel » à la démocratie libérale. Évidemment, le mensonge est d'abord au cœur de la dictature, et donc du régime de la RDA. Il fallait mentir au travail, à l'école, pour cacher ses idées, ses sympathies, ses préférences, l'origine d'objets interdits tels que jeans, disques de jazz ou de rock, livres. Il fallait mentir sur les programmes de télévisions qu'on regardait, ou pas, sur ce que disaient les parents, les amis. Le mensonge par omission était le plus fréquent. Ne pas dire, c'est aussi parfois - mentir.

La trahison, proche parente du mensonge, était un élément constitutif du régime, qui comme nous le savons reposait sur un réseau impressionnant non seulement d'espions officiels, mais aussi de membres non officiels de la fameuse Stasi (Staatssicherheit), Sécurité d'État. Après la fin de ce régime, il était logique que la soif de vérité anime un très grand nombre de citoyens, et que les débats et révélations sur qui avait menti, qui avait trahi, sur les implications du mensonge et des révélations prennent une immense place dans les médias, et dans la littérature.

La recherche de la vérité, et le fait de révéler tous les mensonges passés, de démasquer les menteurs, va en effet passionner un grand nombre de citoyens après 1990. Les conséquences des révélations, dans bien des cas dramatiques, vont occuper le devant de la scène pendant des années, et c'est tout le mérite de la démocratie

17 Voir par exemple Helmut Koopmann, «Die Wirklichkeit der Kopfgeburten. Zu Brigitte Burmeisters Unter dem Namen Norma », in Hans-Jörg KNOBLoch, Helmut Koopmann (dir), Der ,gesamtdeutsche' Roman seit der Wiedervereinigung, Tübingen, Stauffenburg Verlag, 2003, p. 99-117, ainsi que: Blé Richard Lorou, «Utopieentwürfe als Lebensalternative. Unter dem Namen Norma als Hymne auf die Revolution? », in Id., "Erinnerung entsteht auf neue Weise ». Wende und Vereinigung in der deutschen Romanliteratur, Kiel, Ludwig, 2003, p. 150-167.

18 Thomas Brussig, Helden wie wir, Berlin, Verlag Volk und Welt, 1995. Traduction française: Le complexe de Klaus, Paris, Albin-Michel, 1998.

19 Ingo Schulze, Simple Storys, Berlin, 1995. Traduction française: Histoires sans gravité, Paris, Fayard, 1999. 
allemande que d'avoir très rapidement permis aux victimes d'avoir accès à la vérité, c'est-à-dire à leurs dossiers secrets (archives de la Stasi, Gauck-Behörde). Recherche de vérité et conséquences des révélations vont donner matière à un certain nombre d'œuvres, dont elles formeront le cœur, ou le moment déclencheur (on peut penser à La Vie des Autres ${ }^{20}$, ou à Good Bye Lenin ${ }^{21}$, pour n'envisager que deux des films les plus célèbres sur la période).

\section{Une société malade de la vérité}

Dans le roman de B. Burmeister, c'est au contraire autour d'un mensonge que va s'articuler toute l'intrigue. Et ce mensonge sera camouflé, présenté comme révélation, comme LA vérité, celle que tout le monde attend.

Le roman, écrit à la première personne, se divise en deux parties de longueur inégale ( $2 / 3-1 / 3$ environ). Leur titre, «Am 17. Juni » - «Am 14. Juli », les place chacune dans une relation plus ou moins souple avec l'Histoire. En réalité, le roman joue en permanence sur les trois niveaux, le passé, le présent, l'avenir, à travers notamment un jeu subtil avec les temps et les modes, que nous ne pouvons pas étudier ici. Le 17 juin fait référence au 17 juin 1953, révolte qui part de Berlin pour s'étendre dans le reste de la RDA et qui sera réprimée comme « putsch contrerévolutionnaire ». C'est aussi, dans l'économie du roman, la journée du 17 juin 1992, une journée que la narratrice va dérouler dans cette première partie, de l'aube à la nuit, mêlant souvenirs, rêves, observations de ce qui se passe autour d'elle aujourd'hui (les habitants de son immeuble) et références au passé proche ou lointain qui éclaire le présent.

Marianne Arendts, la narratrice, est traductrice. Elle travaille chez elle, dans un appartement situé au dernier étage d'un de ces immeubles avec cours et arrièrecours, ces « Mietskasernen » de Berlin-Mitte. Elle traduit une biographie de SaintJust $^{22}$ et nous comprenons également après quelques pages qu'elle écrit des lettres, réelles ou imaginaires, adressées à son mari, Johannes, parti travailler à l'Ouest « quelque part entre Mannheim et Heidelberg ». Les autres protagonistes sont Max, amant occasionnel et représentant surtout la tentative de vivre une nouvelle utopie (bars alternatifs, création d'une commune à Meißen) et Norma, l'amie fantasque et solide à la fois, qui va se révéler un véritable point d'ancrage pour Marianne, tout en remettant en permanence en question sa manière de vivre. Norma, rencontrée lors de cette nuit d'euphorie et de liberté retrouvée, la nuit du 9 novembre 1989.

Tout le roman, et en particulier cette première partie, confronte le passé proche (les deux années qui viennent de s'écouler depuis cette nuit-là) et le présent (juin 1992), la vie avant l'unification et aujourd'hui, décrit les conséquences de cette révolution, du changement radical de système sur les habitants du microcosme autour de Marianne: un immeuble et quelques rues du quartier de Berlin-Mitte.

\footnotetext{
${ }^{20}$ Das Leben der anderen, réalisateur Florian Henckel von Donnersmarck, Allemagne, 2006.

${ }^{21}$ Good Bye, Lenin!, réalisateur Wolfgang BECKER, Allemagne, 2003.

${ }^{22}$ En 1989, B. Burmeister traduit elle-même l'ouvrage de Bernard Vinot, Saint-Just, sous le pseudonyme de Franziska SaAlburg: Bernard Vinot, Saint-Just, Paris, Fayard, 1985.
} 
La seconde partie tourne autour du mensonge, de son irruption dans la vie des protagonistes, et de ses conséquences. Comme pour la première partie, le récit va et vient entre passé, proche ou plus lointain, et présent. Dans les deux premiers chapitres, le présent: le retour en train de la narratrice chez elle - et le passé proche: un récit linéaire de la période qui va du 9 novembre 1989 à la période de la narration. Le lecteur comprend que Marianne est de retour après un séjour de trois semaines en Allemagne de l'Ouest, là où son mari Johannes vit et travaille, et que la rupture est consommée. L'amitié avec Norma semble également en danger. Parallèlement, tous les éléments qui seront utilisés pour le mensonge sont mis en place, comme des indices à la seule adresse du lecteur, bien sûr. La suite va mettre en scène le contexte du mensonge, puis le mensonge lui-même, enfin ses conséquences, qui seront déroulées dans un récit que Marianne fait à Norma, qui a finalement rejoint la narratrice dans son appartement. Le dernier chapitre enfin thématise le lien entre l'exigence absolue d'un monde meilleur et la Terreur, dans la personne de Saint-Just. La pureté, la recherche intransigeante de la vérité sont ainsi démasquées comme portant en germe l'inhumanité ${ }^{23}$. L'amitié est au contraire célébrée (en parodiant le projet de Saint-Just) par un pacte entre Norma et Marianne, sous le regard de Max, garant du lien qui représente la nouvelle utopie.

Le mensonge de Marianne, camouflé ou revendiqué comme aveu, est L'Aveu de l'époque, donc le pire mensonge possible: lors d'une fête que Johannes organise pour célébrer son intégration réussie à l'Ouest, et le choix que sa compagne a fait, croit-il, de quitter l'Est pour s'installer avec lui, Marianne va révéler son passé à l'une des convives. Elle va avouer qu'elle a été IM, informatrice de la Stasi, « Sous le nom de Norma ». Lorsque Johannes apprendra ce mensonge, qu'il interprètera comme une manière raffinée de dire la vérité, ce sera la rupture.

Avant d'analyser le mécanisme du mensonge de Marianne, et la manière dont elle va se servir d'un mensonge pour reconquérir son identité, il faut rappeler comment naît ce mensonge, à partir du statut particulier de la vérité, ou de la révélation de la vérité, dans une situation donnée. Pour mentir, il faut être deux - ou plus.

En amont de l'infernal déroulement du mensonge se situe en effet le récit du suicide d'une des habitantes de l'immeuble, Margarete Bauer, une victime typique du Tournant, du changement radical du système: auparavant employée dans une maison d'édition, Margarete Bauer perd rapidement son emploi après le passage à l'économie de marché, n'en retrouve pas d'autre malgré tous ses efforts, voit son fils « prendre la fuite » devant la dégringolade sociale de sa mère. Sa solitude, complète après la rupture avec son compagnon des dernières années, et surtout l'impossibilité de donner un nouveau sens à sa vie dans ce monde totalement nouveau qu'elle doit affronter, comme bien d'autres, explique - peut-être - son geste: Margarete Neumann se donne la mort en se jetant du dixième étage.

${ }^{23}$ V. Jankélévitch, dans sa réflexion sur le mensonge, évoque bien cet aspect problématique de l'utilisation de la vérité, lorsqu'il parle d'une véridicité «pire que l'imposture, pire que la calomnie; par exemple quand on dit une vérité qui tue, et qu'on la dit pour tuer ». JANKÉLÉviTCH, « Les vertus et l'amour », p. 188. 
La narratrice, qui n'avait plus guère de relations avec cette voisine, éprouve néanmoins le besoin de lui rendre un dernier hommage, et tente de convaincre son amie Norma de l'accompagner aux obsèques.

Celle-ci refuse catégoriquement. La raison du refus, et la dispute qui s'ensuit entre les deux amies, s'inscrit dans la problématique dramatique du mensonge, de la révélation, et de la rumeur (entre vérité et mensonge: «Il n’y a pas de fumée sans feu», comme le dit Norma un peu plus tard ${ }^{24}$ ). Bouleversée par les conséquences de la recherche impitoyable de la vérité - qui est peut-être une calomnie, une rumeur à laquelle on ajoute foi à cause de cette atmosphère où la révélation se met à prendre une valeur absolue - la narratrice va pour elle-même faire le choix du mensonge, qui devient sa manière de résister.

\title{
À l'origine du mensonge de Marianne: les conséquences de la recherche de la vérité
}

Car voici comment les habitants de l'immeuble d'à côté (ceux de son immeuble ont d'autres interprétations) expliquent le suicide de Margarete Bauer:

\begin{abstract}
Und im Nachbarhaus, wo Norma wohnt, hatte man das Wesen durchschaut, sein lange gehütetes Geheimnis aus zwei Buchstaben aufgedeckt, natürlich das, jetzt kam alles heraus, ans volle Licht der Wahrheit ${ }^{25}$, und das vertrugen manche nicht, tragisch, aber irgendwo gerecht, Schuld und Sühne, nur so gerieten die Dinge wieder ins Lot, doch, das musste sein, würden Sie denn ein Haus bauen auf sumpfigem Grund, na also, und die Akten lügen nicht, warum sollten sie. ${ }^{26}$
\end{abstract}

L'acte de Margarete (son suicide) est lu comme un aveu. La révélation de son secret, deux lettres, IM. La vérité est présentée comme un objectif absolu, nécessaire pour qu'enfin règne l'ordre, les uns sont coupables, les autres innocents, les uns seront châtiés, les autres réhabilités, et cette étape est décrite comme indispensable pour l'établissement de la nouvelle Allemagne (on ne peut pas construire sur un sol marécageux). Et peu importe si cette recherche fait des victimes, y compris parce que la prétendue vérité n'est qu'une calomnie, ou une rumeur.

À partir de ce moment du roman, Marianne réalise la violence qui se cache dans ce mot, « vérité », et à quel point la recherche de la vérité est en train de détruire les relations tissées entre amis, voisins, concitoyens. Elle va décider de ne pas se ranger du côté de ceux et celles qui disent ou cherchent la vérité. Puis, elle va trouver le moyen de détruire de l'intérieur cette opposition entre coupables et innocents, juges et condamnés.

\footnotetext{
24 «Von nichts kommt nichts », Norma, p. 60.

${ }_{25}$ C'est nous qui soulignons.

26 Norma, p. 42-23.
} 


\title{
Qui décide de la vérité?
}

\begin{abstract}
Das Gerücht über Margarete Bauer. Norma hatte es mir erzählt wie eine wahre Geschichte, keine Spur von Zweifel oder Abstand. Das störte mich, ich sagte es ihr, sie blieb dabei, ich war gereizt, schließlich wütend. Dass sie so etwas mitmachte! Ein mieses Gesellschaftsspiel, jawohl, wobei Spiel wahrlich das falsche Wort sei, angesichts der verheerenden Folgen für die Betroffenen und letztlich für alle, in einem Klima öffentlicher Verdächtigungen und Denunziationen, und dass ausgerechnet sie... Nun war Norma empört. Wie ich dazu käme, die Enthüllung von Tatsachen, das Verbreiten der Wahrheit Denunziation zu nennen! Mies waren die Spitzel, verheerend die Machenschaften eines Überwachungsapparates ohnegleichen, und wer da die Fronten verwischte, stellte sich auf die falsche Seite, schützte die Täter und verfolgte die Opfer, das sollte mir eigentlich klar sein und mich davon abhalten, Alarm zu schlagen, weil das Kaninchen im Begriff sei, die Schlange zu fressen. O Gott, Norma, die Zeitung lese ich selbst, sagte ich oder sagte es noch verletzender, und wusste doch, dass ich damit angefangen hatte, mich aus dem Vorrat zusammengelesener Ausdrücke und Argumente zu bedienen, Norma dasselbe tat und wir anders gar nicht reden konnten, die eigenen Meinung kein Originaleinfall in noch nie gehörten Sätze, aber eigen der Impuls und die Gründe waren, sich einer bestimmten Meinung anzuschließen oder ihr zu widersprechen. Und dieses Eigene verstand sich keineswegs von selbst, fand ich jetzt. ${ }^{27}$
\end{abstract}

Ce jeu subtil avec le discours indirect libre, caractéristique du roman tout entier, manifeste le terrible engrenage de la rumeur qui est impossible à contrer, mais aussi le mécanisme de l'accusation que l'on prononce, et qui soudainement instaure une limite infranchissable entre les êtres: l'une a tort, l'autre doit avoir raison. C'est pour échapper à cette dichotomie destructrice que la narratrice va utiliser de manière subversive un mensonge à double fond, la mise en scène mensongère d'un aveu.

Marianne part rejoindre Johannes, qui s'est adapté au monde de l'Ouest, qui y réussit, qui espère que sa femme va faire le même choix. Mais pour Marianne, l'Ouest n'est peuplé que d'êtres sûrs d'eux-mêmes et qui derrière une aimable façade ne la considèrent que comme un condensé de stéréotypes.

C'est lors de la fête organisée par son mari que Marianne va passer à l'offensive et choisir le mensonge comme arme. Alors qu'elle semble un peu isolée, une des invitées, Corinna Kling, s'efforce de lui tenir compagnie. Ce qui paraît de la gentillesse, des efforts pour la mettre à l'aise, elle qui ne connaît personne des amis de Johannes, laisse vite transparaître l'arrogance et le sentiment de supériorité. Cette relation inégale est démasquée, sans en avoir l'air, par les réponses de Marianne, qui livre les clichés à la demande, les représentations que les Allemands de l'Ouest se font des Allemands de l'Est: «Et moi qui pensais, dit Corinna, que chez vous, à l'Est, on avait gardé les anciennes habitudes alimentaires, puisque tout était tellement arriéré. Ce qui n'est pas toujours un mal. Par exemple ces merveilleuses... allées bordées d'arbres, dis-je $\mathrm{e}^{28} \gg$.

${ }^{27}$ Ibid., p. 58-59.

28 «Und ich dachte, sagte Corinna, bei Ihnen im Osten hätten sich die alten Essgewohnheiten erhalten, wo doch alles rückständiger war. Nicht immer ein Mangel. Zum Beispiel die wundervollen Alleen, sagte ich. »Ibid., p. 218. 
Pendant que les deux femmes discutent, la fête continue. Et Marianne, qui boit un peu plus qu'il ne le faudrait, va peu à peu glisser dans un mensonge extravagant, mais - et c'est là le centre du roman - qui sera d'autant plus pris au sérieux, et cru, par Corinna, puis par Johannes.

Le mensonge est d'emblée mis en scène comme une révélation: « Il est temps que vous sachiez la vérité à mon $\operatorname{propos}^{29} \gg$. Et la situation de la narration est elle aussi construite avec soin, cette fois à l'adresse du lecteur (dans le récit, également en direction de Norma). La séduction que sa propre narration exerce sur Marianne se répercute, et bientôt se reflète, dans l'intérêt de celle qui écoute, et qui par son écoute va inspirer le mensonge:

Ein Anfang immerhin. Wie es weitergehen sollte, wusste ich nicht. [...] ich war so gut drauf und auf eine Geschichte aus, die ich Corinna erzählen könnte, ihr allein. Also müsste sie mir versprechen, nichts weiterzusagen, niemandem. Also lag nahe, dass ich ihr ein Geheimnis anvertrauen würde. ${ }^{30}$

Il s'agit d'un secret, à ne révéler à personne: Corinna va donc être d'autant plus poussée à croire que ce secret est vrai. Mais c'est aussi un secret qui s'invente au fur et à mesure, grâce à l'écoute tendancieuse de Corinna, à son attente d'être confirmée dans les représentations qu'elle a des habitants de la RDA. L'histoire commence. Marianne brosse le portrait de sa famille, un père communiste, une mère qui adopte l'opinion de son mari, un frère engagé depuis 1953 (il fait partie de la garde d'honneur, lors de la mort de Staline). Elle accumule les signaux, qui pour le lecteur sont autant de clichés, pour Corinna en revanche, autant de petits cailloux sur le chemin qui mène à la révélation du secret.

Tout semble correspondre à ce qu'attend Corinna, qui éprouve visiblement un grand plaisir à ces confidences. Quant à Marianne, les réactions de son auditrice stimulent visiblement son imagination : le flot du récit semble de plus en plus naître de l'écoute qui lui est prêtée. Corinna commence à se sentir mal à l'aise devant l'étalage d'horreurs que lui présente Marianne. Mais ce malaise ne vient pas d'un soupçon sur la vérité de ce que raconte son interlocutrice. Plutôt d'un mélange d'impressions : ce qu'elle entend lui est étranger et en même temps cela la conforte dans ses préjugés. Marianne sent qu'elle doit passer à quelque chose de plus conforme encore aux attentes de Corinna. Et de nouveau, la narratrice fournit au lecteur les indices que Marianne fabrique l'histoire, en fonction de son interlocutrice: «Pour l'histoire familiale, ça suffisait. Maintenant, il fallait qu'on parle d'amour. Et ce n'était pas la seule raison, me disais-je. Il fallait que l'écoute en ait valu la peine ${ }^{31}$. »

C'est alors que Marianne passe aux « aveux », après avoir construit tout un décor, et les personnages secondaires de son récit mensonger. On a affaire à une véritable théâtralisation du mensonge, avec progression, moments retardateurs, et sommets. Tout est en place pour le clou des aveux: les études, la vie triste et sans distractions d'une jeune Allemande de l'Est. Corinna trouve qu'elle y va un peu fort, mais ne

\footnotetext{
29 «Es ist an der Zeit, dass Sie die Wahrheit über mich erfahren. » Ibid., p. 223.

${ }^{30}$ Ibid., p. 224.

31 «Mit der Familienschilderung war es genug. Um Liebe sollte es nun gehen. Und nicht allein darum, fand ich. Das Zuhören sollte sich gelohnt haben. » Ibid., p. 234.
} 
doute toujours pas de la véracité de ce qu'on lui raconte. C'est elle qui propose le terme de « victimes actives » pour qualifier les citoyens de la RDA. Mais oui, c'est tout à fait cela, rétorque Marianne, mais ce n'est pas tout à fait ce qu'attend Corinna. Il va donc falloir livrer une coupable:

Sie verstehen mich, sagte ich, hob mein Glas und trank ihr zu. Und auch ich verstehe Ihr Problem! Wünschen wir uns nicht Eindeutigkeit? Lieben oder Hassen? Wirkliche Opfer können wir bedauern, wirkliche Täter verabscheuen. Was aber mit aktiven Opfern? [...] und es kommt noch schlimmer. ${ }^{32}$

Et voilà révélée la rencontre avec l'officier de la Stasi, dont Marianne tombe amoureuse, et tout ce petit cabinet des horreurs bien connu également de Corinna, les rencontres secrètes, les appartements clandestins, les faux noms, avec, à la fin (comment y échapper?), la trahison. «Je fis ce qu'il exigeait. J'ai espionné, j'ai trahi. Par amour. C'est comme ça que ça s'est passé ${ }^{33}$. »

Marianne elle-même trouve son récit beaucoup trop ennuyeux à partir de ce moment-là, mais Corinna n'a plus besoin d'écouter, elle va livrer elle-même la suite des éléments nécessaires au développement de l'histoire: la grossesse, l'amant qui la force à avorter. Prise dans sa propre interprétation pathétique du prétendu aveu, elle propose de révéler toute l'histoire de Marianne-qui s'y oppose vivement, évidemment.

Le mensonge de Marianne va alors suivre son propre cours, c'est-à-dire provoquer les conséquences dévastatrices déjà évoquées et révéler que Corinna n'est pas uniquement l'auditrice idéale, sympathisant avec Marianne, qu'elle appelle maintenant « victime tragique ». Elle va en effet s'empresser de trahir sa confiance: l'aveu, qui n'était fait qu'à elle seule, elle va le révéler à son tour à son mari, qui en parlera à Johannes - ce qui provoquera la rupture.

Contrairement à l'ironie, le mensonge veut être pris au sérieux ${ }^{34}$. Or, tout au long du récit, de nombreux indices ont été donnés au lecteur, indiquant qu'il avait affaire à un mensonge, ou plutôt à une histoire mensongère. Il s'agit en effet moins de tromper que de mettre à jour, par cette construction, une vérité inavouable, celle de l'image que se font de leurs concitoyens de l'Est les habitants de l'Ouest. D'une part, à plusieurs reprises l'accent a été mis sur le rôle de celle qui écoute pour la progression de l'histoire, faisant de Corinna une co-auteure de celle-ci, qu'elle remplit de ses propres représentations, jusqu'à la fin du récit: « Une terrible affaire, dit-elle. Oui, répondis-je. [...] Je vous remercie quand même de m'avoir écoutée si patiemment. Sans vous, je n'aurais jamais raconté cette histoire ${ }^{35}$.» Le lecteur a d'autre part été alerté par l'aspect énorme du mensonge, les détails invraisemblables s'accentuant vers la fin. Ces détails auraient également dû alerter Corinna. Mais même exagérés, ces éléments semblent correspondre à l'image, visiblement épouvantable, que l'Allemande de l'Ouest se fait de la vie

${ }^{32}$ Ibid., p. 235-236.

33 « Ich tat, was er verlangte. Ich habe bespitzelt und verraten. Aus Liebe. So war das. » Ibid., p. 238.

34 «L'ironie nous dupe en nous tendant la perche. Mais le mensonge, lui, ne veut pas être interprété: il n'est pas fait pour cela! Il prétend être compris au sens grammatique, c'est-à-dire mécompris, et dissimule soigneusement son arrière-pensée. » JANKÉLÉVITCH, « Les vertus et l'amour », p. 205.

35 « Eine schlimme Sache, sagte sie. Ja, sagte ich. [...] Trotzdem danke für Ihr geduldiges Zuhören. Ohne Sie hätte ich die Geschichte nie erzählt. » Norma, p. 244. 
des Allemands de l'Est, ce qui explique que rien ne la choque dans les horreurs que Marianne a racontées sur son propre compte. Marianne avait raison en utilisant un mensonge comme révélateur de la vérité, c'est-à-dire des opinions cachées des uns, à l'Ouest, sur les autres, restés à l'Est, et c'est ce qui la révolte:

«Car jamais, au grand jamais, ma véritable histoire n'aurait rencontré autant de crédulité que ce montage de clichés horribles mais attendus ${ }^{36}$. »

Les conséquences du mensonge se feront sentir lorsque celui-ci, pris pour ce qu'il est d'abord: un mensonge affreux, donc une trahison (Marianne a trahi la confiance de Corinna, qui la croyait et l'écoutait avec sympathie), puis pour la vérité, va arriver aux oreilles de Johannes. La scène de la révélation du mensonge, et de la rupture qui en est l'effet, ne passe pas sous silence la violence que provoque le désir de vérité: «La vérité, cria-t-il, et si tu ne veux pas me la dire, je te frapperai jusqu'à ce que ça sorte $^{37}$ ! »

Mais Johannes ne voudra pas croire la vérité: « J'ai menti à Corinna Kling, je lui ai menti sans vergogne ${ }^{38}$. » Cela, c'est ce que Marianne dit à Norma, et donc au lecteur. Mais à Johannes, elle va essayer d'expliquer le mécanisme de son mensonge:

Die Form war gegeben, sagte ich, der Inhalt ergab sich dann, Schritt für Schritt. [...] Ich war aber nicht allein. Er schüttelte mich wieder: Du willst mir doch nicht weismachen, dass dir ein fremdes Wesen diese Geschichte eingegeben hat? Mehrere, sagte ich, und etliche bekannte, ich selbst natürlich auch, und Corinna mit ihren Fragen, ihrem Verständnis. ${ }^{39}$

Cette logique du mensonge est absolument inacceptable pour Johannes, et il va interpréter cette explication d'une manière totalement opposée: il va prendre le mensonge au pied de la lettre, lire cette histoire compliquée comme la révélation d'une vérité cachée, LA vérité honteuse par excellence, c'est-à-dire, dans ce contexte, comprendre que Marianne a vraiment travaillé pour la Stasi. Son mensonge est devenu la vérité qu'il plaque sur son identité. Ainsi, le mensonge a démasqué Corinna, mais aussi Johannes, alors que Marianne est à la recherche de l'identité équivoque: la sienne. «J'ai donc vidé mon sac, et j’ai révélé et perdu mon identité, dans les deux sens, tu comprends ${ }^{40}$ ? »

Johannes, lui, a basculé dans la certitude. En rejoignant la manière de voir de Corinna, il est définitivement passé de l'autre côté, du côté des juges, des bons, de ceux qui savent et qui ont raison - du côté des Saint-Just. Il reste avec Corinna, du côté des vainqueurs, tandis que Marianne retourne à l'Est.

En replaçant le roman de B. Burmeister dans une continuité avec l'œuvre d'I. Morgner, nous avons voulu montrer qu'une certaine situation historique ne

36 « Denn nie und nimmer wäre meine reale Geschichte auf solche Glaubensbereitschaft gestoßen wie der Zusammenschnitt von erwartungsgemäßen Gruselbildern. » Ibid., p. 252.

37 « Die Wahrheit, schrie er, und wenn du sie mir nicht sagen willst, prügele ich sie aus dir heraus! » Ibid., p. 249.

38 « Ich habe Corinna Kling belogen nach Strich und Faden. » Ibid., p. 250.

39 Ibid., p. 250-251.

40 «Also habe ich ausgepackt, sagte ich, und bin meine Identität losgeworden, im doppelten Sinn, verstehst du? »Ibid., p. 252. 
semble pas laisser d'autre choix que le mensonge ou la mystification pour tenter de rompre les logiques implacables de la domination. Certes, ces logiques perdurent, par-delà la fin des œuvres envisagées, et ne seront brisées que par la modification de la société elle-même, qu'il s'agisse de la domination du masculin sur le féminin ou de celle des vainqueurs de l'histoire sur les perdants du libéralisme. Mais ce que font I. Morgner comme B. Burmeister, c'est revendiquer le mensonge comme arme de libération de la parole des dominés, comme une manière, évidemment limitée, de reconquérir une identité déniée. Aucun des romans évoqués ne se laisse bien sûr réduire au seul mensonge, à moins que l'on n'étende le mensonge à la mystification littéraire. Et c'est un peu ce que suggère B. Burmeister, en choisissant comme nom de code de l'aveu mensonger une formule, "Sous le nom de Norma », où se cache l'anagramme du mot roman. Ce sont dans tous les cas les assignations univoques, les limites bien tranchées entre coupables et victimes, les identités figées autant que les mémoires, qu'il s'agit de remettre en question. Car celles-ci ne peuvent en aucun cas constituer la base d'une communauté d'êtres libres, ni même celle d'une communication sincère. Pour cela, il faut bien passer par autre chose que la proclamation d'une vérité attendue et vitrifiée. Dépasser les oppositions de genre, et passer à l'amitié féminine: chez I. Morgner comme B. Burmeister, c'est bien l'horizon ultime de l'utopie. 